\title{
Study of Efficacy and Safety of Platelet Rich Plasma Therapy Combined Withmicroneedling on Acne Scars
}

\author{
Harmeet Kaur ${ }^{1}$, Sachin Agarwal' ${ }^{2}$, Amarjeet Singh ${ }^{3}$, Akhil Kumar Singh ${ }^{4}$, Sagar ${ }^{5}$, Parth $^{6}$ \\ ${ }^{1}$ Post graduate, ${ }^{2}$ Professor, ${ }^{3}$ Asisstant Professor, Department of Dermatology, ${ }^{4}$ Associate Professor, Department of \\ Dermatology, Sarawathi institute of Medical Sciences Hapur(U.P)
}

\begin{abstract}
Background: - Acne Vulgaris is an extremely common disorder.More than ninety percent of the patients with acne vulgaris suffer scarring leading topsychological impact and cosmetic disfigurement after healing of their acne lesions.Atrophic acne scars are more common and difficult to treat .These can be sub-classified into icepick, rolling and box car depending on theirwidth, depth and shape. Microneedling device performs acne scar remodelling viacollagen induction therapy. Autologous platelet-rich plasma therapy combined withmicroneedling is a new adjuvant treatment modality of the wound healing cascadebased on the body self-healing mechanisms.
\end{abstract}

Objective-: Assesment of safety and efficacy ofautologus platelet rich plasma therapy combined with microneedling on atrophic acne scars.

Methods: 46 patients with acne scars of 18-25 years age group underwentthree consecutive sittings of microneedling combined with PRP procedure, conducted at an interval of 4 weeks followed by a last assessment done after 3 months of final sitting.

The lesions were classified on Goodman's qualitative scale and serial photography.

Results: Two independent dermatologists performed evaluation and follow up at subsequent visits. Majority (34) patients with good response (improvement by Grade 1) constituting to $(73.91 \%)$ in total. There was an overall improvement in $86.9 \%$ of cases without any serious complications.

Conclusion: - Autologus PRP combined with Microneedling is an effective modality in enhancing clinical outcomes and overall cosmetic appearence of acne scars.

Keywords: acne ,comedones, derambrasion, micorneedling.

\section{Introduction}

As per global burden of skin disease study data, Acne comprises one of the three most common skin diseases. Acne is an inflammatory chronically persisting disease of the pilosebaceous units. It has wide spectrum

\section{Corresponding Author:}

Dr Sachin Agarwal

Professor, Department of Dermatology

Saraswathi Institute of Medical Sciences, Hapur (U.P)

Harypol278@gmail.com of presentation from open and closed comedones, erythematous papules and pustules, nodules, pseudocysts and deep pustules. It usually starts in adolescence and by the mid-twenties undergoes resolution ${ }^{1}$.Its peak distribution seen in prevalence and severity between 14- 17 years aged females, when $40 \%$ are affected, and between 16 and 19 years in males, when $35 \%$ are affected ${ }^{2}$.

Resolution seems to occur in two ways, either as resolution of individual lesions or resolution of the disease as a whole. Post inflammatory skin pigmentation 
and scarring are common residuals. It has been observed in a clinical trial that some degree of scarring is observed in as many as ninety five percent of acne patients. It has been shown that individual sebaceous glands function at different rates in all Acne patients ${ }^{3}$. Resolution may be achieved with specific changes in these Acne-prone hypersecretory glands related to cytokine activity in glands ${ }^{4}$.

The Acne scars are classified broadly as macular, atrophic, hypertrophic or keloidal scars. Further atrophic Acne scar divided into; Ice pick, Rolling and box scars ${ }^{8}$. Atrophic Acne scars seem to occur more commonly in population than keloidal and hypertrophic scars with a ratio 3: 1 . The ice pick type constituted $(60 \%-70 \%)$ of total scars, the box scar $(20 \%-30 \%)$, and rolling scars $(15 \%-25 \%)$ as seenin one clinical trial' ${ }^{2}$. Injury due to the skin inflammation initiates a cascade of wound healing events?.

The importance of reducing the duration and intensity of the inflammation lies in stressing on the importance of the Acne treatment. A number of treatments modalities are available to reduce the overall visibility/appearance of scars.

Micro needling represents as one of the important existing treatment modalities for Acne scars, stretch marks, wrinkles, and facial rejuvenation. It is a rolling device which is embedded with multiple small needles which is used in procedure. The device is rolled multiple times on desired site; for required depth causing micro injury to dermis ${ }^{10}$.

The phase one inflammation starts immediately after the procedure followed by fibroblasts invasion. The wound closure function of fibroblasts is performed by migration to the point of invasion for wound healing. It also leads to stimulation of endothelial cells which causes neo- angiogenesis. The natural tissue remodeling further continues for 8 weeks to 1 year $^{11,12}$. Atrophic acne scars ${ }^{13,14,15}$ can be effectively managed by Micro needling. The technique of Micro needling is easy, cost effective, and associated with fewer side effects and short downtime period.

Autologous platelet-rich plasma (PRP) therapy is one of the latest upcoming techniques in the field of dermatology. It was originally started and used as an autologous transfusion component after an openheart operation to avoid homologous blood product transfusion ${ }^{16}$.

The optimal PRP platelet concentration after having $300-700 \%{ }^{17}$ enrichment should be more than 10 lakhs platelets/ $\mu 1$. It contains high concentrations of platelet derived growth factors.

Higher amounts of platelet derived growth factors present in concentrate can make it suitable to use in several other therapeutic indications such as treatment of androgenic alopecia, Acne scars, hair transplantation graft survival, skin rejuvenation, better wound healing, face lifting and several other cosmetic procedures ${ }^{18-21}$.

Platelets on activation can release several growth factors and cytokines like platelet-derived growth factors (PDGF), transforming growth factors (TGF), vascular endothelial growth factor (VEGF), Insulin-like growth factor (IGF), epidermal growth factor (EGF), and interleukin (IL)-1. Fibroblasts are the cells that are activated by TGF-beta ${ }^{22}$.

\section{Materials and Method}

The present study was carried out in the Department of Dermatology, Venereology and Leprosy, Saraswathi Institute of Medical Sciences, Hapur. In this prospective, time-bound study, 50 patients diagnosed with atrophic acne scars were included from October 2017 to February 2019. Prior approval was taken from medical ethical and scientific committee of the hospital. Out of fifty patients four patients left treatment in between and did not appear for follow up, so study was completed on 46 patients in given time frame.

\section{Selection of Patients}

\section{- Inclusion criteria}

$\varnothing$ Patients with grade 2 to 4 Atrophic acne scars, classified on the basis of Goodman's qualitative classification.

$\varnothing \quad$ No active acne lesions.

\section{- Exclusion criteria}

$\varnothing \quad$ Positive history of keloidal tendency.

$\varnothing \quad$ Patients having history of bleeding diathesis or 
platelets related disorders or any anticoagulant therapy,.

$\varnothing \quad$ History of major surgery in past six months.

$\varnothing \quad$ Presence or history of any acute infection on face like herpes, folliculitis etc.

$\varnothing$ Patients of HIV, HBsAG, or any other chronic illness.

$\varnothing \quad$ Pregnant females.

Final results for clinical improvement were made using following parameter:

Mean scores of Pre and Post treatment Goodman's qualitative scores were calculated for right and left halves and percentage of improvement was calculated for both the halves combinedly. Paired t test was applied among pre and post treatment scores of right and left halves.

\section{Procedure-}

\section{Preparation of Platelet Rich Plasma (PRP)}

$3 \mathrm{ml}$ of anticoagulant (ACD) was taken in $20 \mathrm{ml}$ syringe from anticoagulant bag.Patients were kept supine in stable position, and $17 \mathrm{ml}$ of venous blood was withdrawn in above syringe. Gentle shaking of the Syringe was done for 5 times for proper mixing of anticoagulant with blood.

- Initially, two sterile $10 \mathrm{ml}$ tubes were taken and blood was transferred to each tube.

- All blood samples were processed at blood bank for preparation of platelet rich plasma. Standard REMI centrifugation machine was used for centrifugation process.

- All blood samples were processed at blood bank for preparation of platelet rich plasma. Standard REMI centrifugation machine was used for centrifugation process.

- First centrifugation was done after $30 \mathrm{~min}$ from blood sampling at 1500 Revolutions per minute (rpm) for $5 \mathrm{~min}$.

- Three layers were formed in each tube; i.e. plasma, buffy coat and red cell sediments.
- Plasma and part of buffy coat was separated in separate sterile tube, and red cell sediments were discarded.

- The resultant solution was further centrifuged at $2300 \mathrm{rpm}$ for $15 \mathrm{~min}$.

- Two layers of plasma were formed; dense layer of platelets at the bottom and clear fluid layer on the top. Most of the clear fluid (platelet poor plasma) was removed, leaving behind small solution which is then gently wobbled to form platelet rich plasma.

- Platelet count assessment was performed in automated platelet counter machine in pathology department before each session for desired platelet number achievement.

\section{The Instrument}

- The dermaroller used for microneedling procedure in the present study had $1.5 \mathrm{~mm}$ long needles similar to the other studies conducted in India.

o After application of topical anaesthesia, PRP was injected intradermally into acne scars using insulin syringe followed by microneedling performed 6-8 times ineach direction in four quadrants .

Follow-up

th Patients were observed for follow up on 2 and 30 day post procedure. On 30 day the procedure was repeated as per above guidelines.

\section{Results}

- Total 3 sessions of microneedling were performed at monthly interval. Final assessment of improvement was done for three months at monthly intervals after completion of treatment for each patient

\section{Statistical Analysis}

The data was coded and entered into Microsoft Excel spreadsheet. Analysis was done using SPSS version 20 (IBM SPSS Statistics Inc., Chicago, Illinois, USA) Windows software program. Descriptive statistics included computation of percentages, means and standard deviations. Chi-square test used for qualitative data whenever two or more than two groups were used to compare. Level of significance was set at $P \leq 0.05$. 
DISTRIBUTION OF CASES ACCORDING TO GRADES BEFORE TREATMENT

\begin{tabular}{|l|c|c|}
\hline Grade & Frequency & Percent \\
\hline Grade 4 & 18 & $39.1 \%$ \\
\hline Grade 3 & 23 & $50.0 \%$ \\
\hline Grade 2 & 5 & $10.86 \%$ \\
\hline Grade 1 & 0 & $0 \%$ \\
\hline Total & 46 & 100.0 \\
\hline
\end{tabular}

Comparison Of Proportions According To Grades Before The Treatment And After Third Sitting

\begin{tabular}{|c|c|c|c|c|c|c|c|}
\hline & & & \multicolumn{4}{|l|}{ Score } & \multirow{2}{*}{ Total } \\
\hline & & & Grade 1 & Grade 2 & Grade 3 & Grade 4 & \\
\hline \multirow{4}{*}{ Time interval } & \multirow{2}{*}{$\begin{array}{l}\text { Before } \\
\text { treatment }\end{array}$} & $\mathrm{N}$ & 0 & 5 & 23 & 18 & 46 \\
\hline & & $\%$ & $0.0 \%$ & $10.9 \%$ & $50.0 \%$ & $39.1 \%$ & $100.0 \%$ \\
\hline & \multirow{2}{*}{$\begin{array}{l}\text { After } 3 \\
\text { sitting }\end{array}$} & $\mathrm{N}$ & 1 & 17 & 21 & 7 & 46 \\
\hline & & $\%$ & $2.2 \%$ & $37.0 \%$ & $45.7 \%$ & $15.2 \%$ & $100.0 \%$ \\
\hline \multirow{2}{*}{ Total } & & $\mathrm{N}$ & 1 & 22 & 44 & 25 & 92 \\
\hline & & $\%$ & $1.1 \%$ & $23.9 \%$ & $47.8 \%$ & $27.2 \%$ & $100.0 \%$ \\
\hline
\end{tabular}

Distribution of cases according to the grading before the treatment, after 3 sittings and after 3 months of final sitting

\begin{tabular}{|c|c|c|c|c|c|c|c|}
\hline & & & \multicolumn{4}{|l|}{ Score } & \multirow{2}{*}{ Total } \\
\hline & & & Grade 1 & Grade 2 & Grade 3 & Grade 4 & \\
\hline \multirow{6}{*}{$\begin{array}{l}\text { Time } \\
\text { interval }\end{array}$} & \multirow{2}{*}{ Before treatment } & $\mathrm{N}$ & 0 & 5 & 23 & 18 & 46 \\
\hline & & $\%$ & $0.0 \%$ & $10.9 \%$ & $50.0 \%$ & $39.1 \%$ & $100.0 \%$ \\
\hline & \multirow{2}{*}{ After 3 sitting } & $\mathrm{N}$ & 1 & 17 & 21 & 7 & 46 \\
\hline & & $\%$ & $2.2 \%$ & $37.0 \%$ & $45.7 \%$ & $15.2 \%$ & $100.0 \%$ \\
\hline & \multirow{2}{*}{ After 3 month } & $\mathrm{N}$ & 5 & 25 & 14 & 2 & 46 \\
\hline & & $\%$ & $10.9 \%$ & $54.3 \%$ & $30.4 \%$ & $4.3 \%$ & $100.0 \%$ \\
\hline
\end{tabular}


Overall out of 46 cases, There were 6 cases $(13.04 \%$ ) 4 cases of Grade 4 improved to Grade 2 and 2 cases of Grade 3 improved to Grade 1 with an excellent response. There were majority (34) patients with good response (improvement by Grade 1) constituting to (73.91\%) in total that comprises of 12 cases with Grade 4 improved to Grade 3, 19 cases with Grade 3 improved to Grade 2 and 3 cases with Grade 2 improved to Grade 1. Hence, there was an overall improvement in $86.9 \%$ of cases without any serious complications like milia formation or post inflammatory hyperpigmentation. There were 6 cases (13\%) that is 2 cases each of Grade 4, 3 and 2 in which there was no improvement.

\section{Discussion}

Acne scar management has been a challenging task and focus of interest of Dermatologists for several decades. Because of the work of various investigators, remarkable advances have been made to revise atrophic acne scars namely ice pick, rolling or superficial, and boxcar or depressed fibrotic scars. In present study an attempt has been made to prove the efficacy of micro needling combined with autologous platelet rich plasma therapy for atrophic acne scars which is a cheap and an effective modality to treat atrophic scars.

Out of the total 50 cases recruited, 46 cases completed the study with 3 sittings at an interval for 4 weeks and followed up for 3 months after the final sitting.

\section{Grading System}

In the present study measurement of efficacy of acne scars improvement and their grading was done by Goodman and Baron Grading system. This grading scale was also used in most of the other studies conducted in India and other parts of the World (Srivastava $\mathrm{R}$ et al 2016, Pandey A et al 2015, Dogra S et al 2014, Alam M et al 2014, Asif et al while only Fabbrocini et al in 2014 used the Global Aesthetic improvement scale.

\section{Conclusion}

Microneedling combined with Autologous Platelet Rich Plasma therapy is an excellent option for the treatment of atrophic acne scars with good clinical outcomes, though recently ablative and non-ablative lasers have been introduced but they have their own limitations. The down time (erythema) may be present for months together with ablative lasers. Hence, it is a low cost, well tolerable and a very simplified day care procedure with minimal side effects and complications.

\section{Acknowledgment-}

The authors are thankful to chairman and managing member of Saraswathi institute of medical sciences, Hapur, U.P for their encouragement.

Ethical Clearance- Taken from ethical committee of institute

\section{Source of Funding- Self}

Conflict of Interest $-\mathrm{Nil}$

\section{References}

1. Adityan B, Kumari R, Thappa DM. Scoring systems in acne vulgaris. Indian Journal of Dermatology, Venereology and Leprology 2009; 75 (3): 323-6.

2. Bhate, K; Williams, HC. Epidemiology of acne vulgaris. The British journal of dermatology 2013; 168 (3): 474-85.

3. Barnes LE, Levender MM, Fleischer AB Jr, Feldman SR. Quality of life measures for acne patients. Dermatologic Clinics 2012; 30 (2): 293300.

4. Benner N, Sammons D. Overview of the treatment of acne vulgaris. Osteopathic Family Physician 2013; 5 (5): 185-90.

5. James WD. Acne. New England Journal of Medicine 2005; 352 (14): 1463-72.

6. Levy, LL; Zeichner, JA. Management of acne scarring, part II: a comparative review of nonlaser-based, minimally invasive approaches. Am J ClinDermatol 2012; 13 (5): 331-40.

7. Bellafill for acne scars. The medical letter on drugs andtherapeutics 2015;57(1471):93-94.

8. Epstein RE, Spencer JM. Correction of atrophic scars withartefill: an open-label pilot study. J Drugs Dermatol2010;9(9):1062-1064

9. Erickson JR,Echeveerri K. Learning from regeneration research organisms:the circuitous road to scar free wound healing.DevBiol.2018 Jan 15;433(2):144-54

10. Singh A,YadavS.Microneedling:Advances and 
widening horizons.IndianDermatol online J .2016 Jul-Aug;7(4):244-54.

11. Moghadam

A, FraimaiMM, Soroury S, EbrahimiNS. Hypermongone $\mathrm{C}$ accelerates wound healing through the modulation of inflammatory factors and promotion of fibroblast migration.Molecules 2019 May ;24(10)

12. LinPH,Sermersheim,LiH,LeePH,SteinbergSM. Zinc in wound healing modulation.Nutrients,2018 Jan;10(1):16.

13. El-Domyati M, Barakat M, Awad S, Medhat W, ElFakahany H, Farag H. Microneedling Therapy for Atrophic Acne Scars: An Objective Evaluation. J ClinAesthetDermatol. 2015;8(7):36-42.

14. El-Domyati M, Barakat M, Awad S, Medhat W, ElFakahany H, Farag H. Microneedling Therapy for Atrophic Acne Scars: An Objective Evaluation. J ClinAesthetDermatol. 2015;8(7):36-42.

15. Minh PPT, Bich DD, Hai VNT, et al. Microneedling Therapy for Atrophic Acne Scar: Effectiveness and Safety in Vietnamese Patients. Open Access Maced J Med Sci. 2019;7(2):293-297. Published 2019 Jan 29.

16. Carless PA, Rubens FD, Anthony DM, O'Connell D, Henry DA. Platelet-richplasmapheresis for minimisingperi-operative allogeneic blood transfusion. Cochrane Database of Systematic Reviews. 2003;(2)

17. Maria Rosaria De Pascale, Linda Sommese, Amelia Casamassimi, and Claudio Napoli,Platelet derivatives in regenerative medicine: an update. Transfusion Medicine Reviews, 2014.

18. Okereke UR, Simmons A, Callender VD. Current and emerging treatment strategies for hair loss in women of color. Int $\mathrm{J}$ WomensDermatol. 2019;5(1):37-45.

19. Aldoori N., Dobson K., Holden C.R., McDonagh A.J., Harries M., Messenger A.G. Frontal fibrosing alopecia: Possible association with leave-on facial skin care products and sunscreens; a questionnaire study. Br J Dermatol. 2016;175(4):762-767.

20. Gentile P, Garcovich S. Advances in Regenerative Stem Cell Therapy in Androgenic Alopecia and Hair Loss: Wnt pathway, Growth-Factor, and Mesenchymal Stem Cell Signaling Impact
Analysis on Cell Growth and Hair Follicle Development. Cells. 2019;8(5):466

21. Sulzberger, MB and witten, VH:Hormone and acne vulgaris.Med. Clin N Amer.1951;35:373-390.

22. Fabian Cieplik, Laura Tabenski, Karl-Anton Hiller, Gottfried Schmalz, Wolfgang Buchalla and Michael Christgau, Influence of autogenous platelet concentrate on combined GTR/graft therapy in intra-bony defects: A 13-year followup of a randomized controlled clinical split-mouth study, Journal of Clinical Periodontology, 2018;45, 3, 382-91.

23. NourianDehkordi A, MirahmadiBabaheydari F, Chehelgerdi M, RaeisiDehkordi S. Skin tissue engineering: wound healing based on stem-cellbased therapeutic strategies. Stem Cell Res Ther. 2019;10(1):111.

24. Connolly D, Vu HL, Mariwalla K, Saedi N. Acne Scarring-Pathogenesis, Evaluation, and Treatment Options. J ClinAesthetDermatol. 2017;10(9):1223.

25. A. M. Layton, C. A. Henderson, and W. J. Cunliffe. A clinical evaluation of acne scarring and its incidence. Clinical and Experimental Dermatology. 1994;. 19(4): 303-308.

26. S. Z. Ghodsi, H. Orawa, and C. C. Zouboulis, "Prevalence, severity, and severity risk factors of acne in high school pupils: a community-based study," Journal of Investigative Dermatology, vol. 129, no. 9, pp. 2136-2141, 2009.

27. Cotterill JA, Cunliffe WJ (1997) Suicide in dermatologic patients. Br J Dermatol 137: 246-250.

28. Gupta, M.A. and Gupta, A.K. Depression and suicidal ideation in dermatology patients with acne, alopecia areata, atopic dermatitis and psoriasis. $\mathrm{Br} \mathrm{J}$

29. Lynn DD, Umari T, Dunnick CA, Dellavalle RP. The epidemiology of acne vulgaris in late adolescence. Adolesc Health Med Ther. 2016;7:1325.

30. Makrantonaki E, Ganceviciene R, Zouboulis C. An update on the role of the sebaceous gland in the pathogenesis of acne. Dermatoendocrinol. 2011;3(1):41-49. 